\title{
Excitation energy transfer in native and unstacked thylakoid membranes studied by low temperature and ultrafast fluorescence spectroscopy
}

\author{
C. D. van der Weij-de Wit · J. A. Ihalainen • \\ R. van Grondelle · J. P. Dekker
}

Received: 22 August 2006/Accepted: 2 March 2007/Published online: 28 March 2007

(C) Springer Science+Business Media B.V. 2007

\begin{abstract}
In this work, the transfer of excitation energy was studied in native and cation-depletion induced, unstacked thylakoid membranes of spinach by steady-state and time-resolved fluorescence spectroscopy. Fluorescence emission spectra at $5 \mathrm{~K}$ show an increase in photosystem I (PSI) emission upon unstacking, which suggests an increase of its antenna size. Fluorescence excitation measurements at $77 \mathrm{~K}$ indicate that the increase of PSI emission upon unstacking is caused both by a direct spillover from the photosystem II (PSII) core antenna and by a functional association of light-harvesting complex II (LHCII) to PSI, which is most likely caused by the formation of LHCII-LHCI-PSI supercomplexes. Time-resolved fluorescence measurements, both at room temperature and at $77 \mathrm{~K}$, reveal differences in the fluorescence decay kinetics of stacked and unstacked membranes. Energy transfer between LHCII and PSI is observed to take place within $25 \mathrm{ps}$ at room temperature and within $38 \mathrm{ps}$ at $77 \mathrm{~K}$, consistent with the formation of LHCII-LHCI-PSI supercomplexes. At the 150-160 ps timescale, both energy transfer from LHCII to PSI as well as spillover from the core antenna of PSII to PSI is shown to occur at $77 \mathrm{~K}$. At room temperature the spillover and energy transfer to PSI is less clear at the $150 \mathrm{ps}$ timescale, because these processes compete with charge separation in the PSII
\end{abstract}

C. D. van der Weij-de Wit $(\bowtie) \cdot$ J. A. Ihalainen ·

R. van Grondelle · J. P. Dekker

Faculty of Sciences, Department of Physics and Astronomy,

Section Biophysics, Vrije Universiteit, De Boelelaan 1081,

Amsterdam $1081 \mathrm{HV}$, The Netherlands

e-mail: chantal@few.vu.nl

J. A. Ihalainen

Physikalisch Chemisches Institut, Universität Zürich,

Winterthurerstrasse 190, Zurich 8057, Switzerland reaction center, which also takes place at a timescale of about 150 ps.

Keywords Energy transfer - LHCII-LHCI-PSI supercomplexes - Low-temperature fluorescence . Spillover · Time-resolved fluorescence · Unstacked thylakoid membranes
Abbreviations
Chl(s) Chlorophyll(s)
LHC Light-harvesting complex
PS Photosystem

\section{Introduction}

In green plant photosynthesis, photosystem I (PSI) and photosystem II (PSII) convert light into chemically stored energy. In the light, they work either in series, with plastoquinone conducting the linear electron transport from PSII to PSI, or PSI is involved in cyclic electron transport with the cytochrome $b_{6} f$ complex. Both photosystems I and II have a peripheral light harvesting-antenna, called LHCI and LHCII, respectively, which absorb light in partially different regions of the visible spectrum. The electron flow between the two photosystems is optimized by a controlled distribution of excitation energy. Redistribution is realized by a process called state transitions, which is based on a migration of 'mobile' LHCII (Murata 1969; Allen and Forsberg 2001; Wollman 2001).

Reversible phosphorylation of LHCII is believed to play an essential role in state-transitions (Dèpege et al. 2003). Upon light illumination favoring PSII a part of LHCII 
becomes phosphorylated and moves from PSII to PSI, i.e. formation of state 2 . A transition to state 1 occurs upon PSI light illumination (or darkness) in which the phosphorylated LHCII becomes dephosphorylated and dissociates from PSI to recombine with PSII again. Phosphorylation and dephosphorylation are under the control of redox sensitive protein kinases and phosphatases present in the photosynthetic membrane (Dèpege et al. 2003). However, a study by Zhang and Scheller (2004) showed that phosphorylation of LHCII is not necessary for its binding to PSI. Photosystem I was found to bind a significant amount of unphosphorylated LHCII in state 2.

Biochemical studies indicated that the PSI-H, -I, -L and -O subunits are involved in the binding of LHCII to PSI (Zhang and Scheller 2004; Lunde et al. 2000; Jensen et al. 2004). The PSI structure of pea (Ben-Shem et al. 2003) shows an empty pocket at the PSI-A, -K and -L units, which was proposed to comprise the docking site for LHCII (Ben-Shem et al. 2004; Dekker and Boekema 2005). Recently, electron microscopy of solubilized thylakoid membranes of Arabidopsis thaliana, prepared either in state 1 or in state 2 , revealed the presence of LHCII-LHCI-PSI supercomplexes, which were more abundant in state 2 (Kouril et al. 2005). The $16 \AA$ projection map could be assigned to a specific association of PSI and one LHCII trimer using the structures published by (Ben-Shem et al. 2003) and (Liu et al. 2004), respectively. The LHCII trimer is attached at a defined position between subunits PSI-A, -H, -L and -K. A possible second binding site for an LHCII trimer is pointed out to consist of the subunits PSI -H, -I, -B and -G. The presence of LHCII was confirmed by excitation spectra of the PSI emission of membranes in state 1 and 2 (Kouril et al. 2005).

The thylakoid membrane is composed of successive stacked regions, the grana, interconnected by single membranes or stroma lamellae. The photosystems are known to be inhomogeneously distributed within the thylakoid membrane, with the grana stacks being rich in PSII and LHCII, whereas the stroma lamellae are richly occupied by PSI (Albertsson and Andreasson 2004). Upon cation-depletion, the grana stacks unfold and a so-called bleb is formed. Unstacking of the thylakoid membrane by cation-depletion is known to increase the PSI fluorescence emission (e.g. Barber 1980; Briantais et al. 1984; Williams et al. 1987; Stoitchkova et al. 2006). Both Briantais and co-workers and Stoitchkova and co-workers assigned this increase to spillover from PSII to PSI, as a result of the induced homogeneous distribution of both photosystems by unstacking. Williams and co-workers showed that the presence or absence of $\mathrm{Mg}^{2+}$ ions hardly influences the organization of PSI-LHCI-LHCII sheets. However, in absence of $\mathrm{Mg}^{2+}$ an increased PSI fluorescence was recorded and LHCII to PSI energy transfer observed by fluorescence emission and excitation spectra, similar to a state 1 to state 2 transition (Kouril et al. 2005). Thus it was concluded that it is not the unstacking of the thylakoid membrane, which initiates the increase of the PSI absorption cross-section, but rather a change in electrostatic forces, which drive LHCII apart, and allow them to functionally associate with PSI.

In this study, we show by low temperature steady-state fluorescence measurements that unstacking of the thylakoid membrane results mainly in energy transfer from LHCII to PSI, but also in direct spillover from the PSII core antenna to PSI. The first process may be the result of the formation of LHCII-LHCI-PSI supercomplexes, similar to a state-transition. Time-resolved fluorescence measurements both at room temperature and $77 \mathrm{~K}$, reveal the time constants of both energy transfer processes.

\section{Materials and methods}

Stacked thylakoid membranes were prepared from fresh, dark-adapted spinach leaves, obtained from local shops. The leaves were depetiolated and ground in a blender in a buffer containing $50 \mathrm{mM}$ Hepes $\mathrm{pH} 7.5,0.4 \mathrm{M} \mathrm{NaCl}$, $2.0 \mathrm{mM} \mathrm{MgCl}, 1 \mathrm{mM}$ EDTA and $2 \mathrm{mg} / \mathrm{ml}$ bovine serum albumin; washed in a buffer containing $50 \mathrm{mM}$ Hepes $\mathrm{pH}$ 7.5, $0.15 \mathrm{M} \mathrm{NaCl}$ and $4.0 \mathrm{mM} \mathrm{MgCl}_{2}$; and diluted in a buffer containing $20 \mathrm{mM}$ Hepes $\mathrm{pH} 7.5,20 \mathrm{mM} \mathrm{NaCl}$, $5 \mathrm{mM} \mathrm{MgCl}_{2}$. Unstacked thylakoid membranes were prepared as above, but $\mathrm{MgCl}_{2}$ was omitted from all buffers, while the grind buffer also contained $4 \mathrm{mM}$ EDTA (Dekker et al. 2002). At room temperature, $1 \mathrm{mM}$ ferricyanide was added to keep the PSII reaction centers open. Photosystem II membranes or BBY particles were isolated from thylakoid membranes as described by (Berthold et al. 1981) with some modifications as described by (Van Leeuwen et al. 1991) and dissolved in the same buffer as the native membranes. Additionally GY particles (named after Ghanotakis and Yocum who where the first ones to isolate this particle), which consist of the PSII core complex and the minor antenna complexes CP29 and CP26 were prepared as in Ghanotakis et al. (1987). The PSI-LHCI complexes were isolated from unstacked spinach thylakoid membranes as described in Dekker et al. (2002) and diluted in a buffer of $20 \mathrm{mM}$ BisTris $\mathrm{pH} 6.5$, $5 \mathrm{mM} \mathrm{MgCl}_{2}$ and $0.06 \%$ of $\beta$-DM. For the low temperature fluorescence measurements, $60 \%(\mathrm{v} / \mathrm{v})$ glycerol was added as cryoprotectant. A nitrogen cryostat (Oxford) was used to cool the samples to $77 \mathrm{~K}$. A helium bath cryostat (Utreks, $4 \mathrm{~K}$ ) was used to cool the samples to $4 \mathrm{~K}$. The isolated membranes were diluted to an optical density less than $0.1 \mathrm{~cm}^{-1}$ at $680 \mathrm{~nm}$ for the steady-state measurements and $0.9 \mathrm{~cm}^{-1}$ for the time-resolved fluorescence measurements. 
Fluorescence emission spectra were measured with a $0.5 \mathrm{~m}$ imaging spectrograph (Chromex 500IS) and a CCD camera (Chromex Chromcam I). The spectral resolution was $0.15 \mathrm{~nm}$. For broadband excitation a tungsten halogen lamp (Oriel) was used with an interference filter transmitting at $420 \mathrm{~nm}$, bandwidth $15 \mathrm{~nm}$.

Fluorescence excitation spectra were measured with a fluorimeter (Jobin Yvon, FL 3-11). The membranes were excited by a Xenon lamp, with both excitation and detection bandwidth $1 \mathrm{~nm}$. The fluorescence was detected at $735 \mathrm{~nm}$.

The time-resolved measurements were performed with a streak camera. Briefly, $486 \mathrm{~nm}$, vertically polarized excitation pulses of $\sim 100$ fs were generated using a Ti:sapphire laser (VITESSE, Coherent St. Clara, CA) with a regenerative amplifier (REGA, Coherent), a double pass optical parametric amplifier (Coherent, OPA) and a Berek compensator. For the room temperature measurements, the excitation light was collimated with a $15 \mathrm{~cm}$ focal length lens, resulting in a focal diameter of $150 \mu \mathrm{m}$ in the sample. The repetition rate was $200 \mathrm{kHz}$ and the pulse energy $1 \mathrm{~nJ}$. The sample was in a $1 \mathrm{~cm}$ polystyrene cuvette and magnetically stirred. For the $77 \mathrm{~K}$ measurements, an unfocussed excitation beam was used with a diameter of $\sim 1 \mathrm{~mm}$. The repetition rate was $50 \mathrm{kHz}$ and the pulse energy $20 \mathrm{~nJ}$. The sample was in a $1 \mathrm{~cm}$ polystyrene cuvette in a nitrogen cryostat (Oxford). Both at room temperature and $77 \mathrm{~K}$, the fluorescence was detected in front-face mode at the magic angle, using a Chromex 250IS spectrograph and a Hamamatsu C 5680 synchroscan streak camera. The streak images were recorded with a cooled, Hamamatsu C4880 CCD camera. The full width at half maximum of the overall time response of the experiments was 28 ps at room temperature and 10 ps at $77 \mathrm{~K}$. In the global analysis, both the instrument response and time dispersion were free parameters of the fit.

The datasets obtained with the streak-camera setup, consist of a two-dimensional image of fluorescence intensity as a function of both time and wavelength. Before analysis, the images were corrected for background and sensitivity of the detection system. These images were sliced up into time traces, which span $4 \mathrm{~nm}$ and was fit with a sum of exponentials with different decay times. The amplitudes of these exponentials as a function of emission wavelength provide Decay Associated Spectra (DAS) where a positive amplitude represents a loss of fluorescence, whereas a negative amplitude reflects a rise of fluorescence. Hence a DAS with a positive amplitude at short wavelengths and a negative amplitude at higher wavelengths represents an energy transfer process, whereas an overall positive spectrum describes either trapping of excitations by one of the reaction centers or a natural fluorescence emission of the pigments (the latter occurs typically at the ns-time scale). For more details, see (Van Stokkum et al. 2004; Holzwarth 1996).

Using the above approach, the overwhelming numbers of dynamic spectral processes, which take place in the system under study, are described with a few essential/ indicative time constants, which describe the main processes of the system. One has to keep in mind, however, that the separation power of the lifetimes is close to a factor of 10 and thus many parallel kinetic processes, which obviously takes place in photosynthetic membranes, may be "lumped", together into one spectrum. Therefore, additional information is required for reliable assignments of the components, which particularly in this case means information from the isolated photosynthetic complexes.

\section{Results and discussion}

\section{$5 \mathrm{~K}$ fluorescence emission}

Figure 1 displays the recorded fluorescence emission spectra at $5 \mathrm{~K}$ upon $420 \mathrm{~nm}$ excitation of both native and unstacked thylakoid membranes, normalized to their optical density in the $\mathrm{Q}_{\mathrm{y}}$ maximum. Four maxima can be clearly discerned in both spectra, i.e., a peak or shoulder at $682 \mathrm{~nm}$, a split band with peaks at 687 and $692 \mathrm{~nm}$ and a broad band with maximum at about $738 \mathrm{~nm}$, which are respectively assigned to LHCII, PSII and PSI. The peak at $687 \mathrm{~nm}$ will cover some PSI-LHCI fluorescence as well (Klimmek et al. 2005). In the spectrum of the native thylakoid membranes, a shoulder at $750 \mathrm{~nm}$ is observed, which is assigned to a collection of vibrational bands of PSII. Upon unstacking of the native thylakoid membranes, the fluorescence emission of both LHCII and PSI are seen

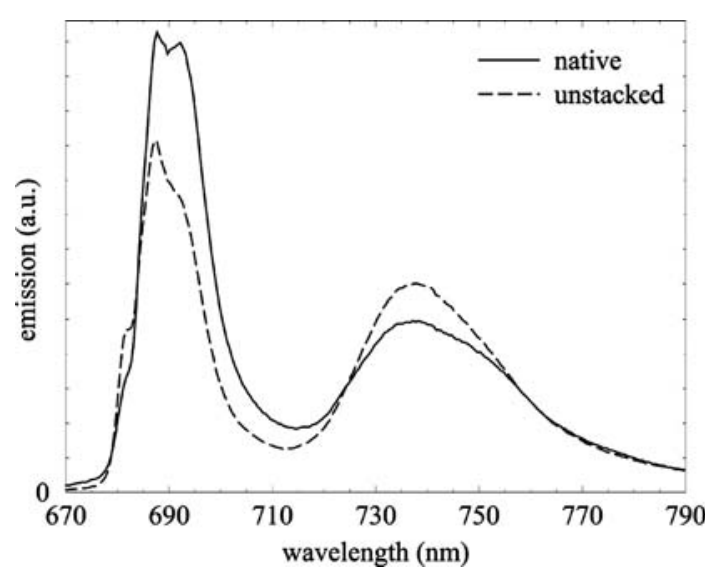

Fig. $15 \mathrm{~K}$ fluorescence emission spectra of native (solid) and unstacked (dash) thylakoid membranes upon $420 \mathrm{~nm}$ excitation. The spectra have been normalized to the respective $Q_{y}$ absorption maxima 
to increase, whereas that of PSII significantly decreased. The relative increase of LHCII fluorescence with respect to PSII is interpreted as a decrease in the energy transfer from LHCII to PSII. This observed reduction in connectivity between LHCII and PSII upon unstacking of the thylakoid membrane, may be caused by a release of LHCII from PSII and/or by a decrease of transversal energy transfer between the membrane layers in a stack (Leibl et al. 1989; Kirchhoff et al. 2004). At the same time, unstacking of the thylakoid membrane is seen to result in an increase of the PSI fluorescence emission. According to the observed decrease in PSII fluorescence at 687 and $692 \mathrm{~nm}$, a decrease of the PSII vibrational band at $750 \mathrm{~nm}$ is also expected to occur. Consequently, the increase in PSI fluorescence at $738 \mathrm{~nm}$ must therefore be significant. The observed increase in PSI emission may be explained by an increased antenna size, realized by spillover from PSII and/ or through the association of released LHCII to PSI. As trimeric LHCII is known to have a high fluorescence yield (Palacios et al. 2002), the relatively small increase in LHCII emission with respect to that of PSI upon unstacking, suggests that in the latter case, the association of released LHCII to PSI must be significant.

\section{$77 \mathrm{~K}$ fluorescence excitation}

To verify whether LHCII leaves PSII for PSI upon unstacking of the thylakoid membrane, $77 \mathrm{~K}$ fluorescence excitation spectra were measured of both the native and unstacked thylakoid membranes. We performed these measurements at $77 \mathrm{~K}$, because at this temperature the fluorescence quantum yield is relatively high for PSI and low for PSII with respect to $5 \mathrm{~K}$ (Andrizhiyevskaya et al. 2002, 2005). Figure 2 shows the recorded $77 \mathrm{~K}$ fluorescence excitation spectra detected at $735 \mathrm{~nm}$ of native and unstacked thylakoid membranes. It has been verified that the contribution of the vibrational fluorescence emission band of free LHCII is small at this detection wavelength-less than $3 \%$ of the emission maximum at $735 \mathrm{~nm}$ - and thus its presence hardly influences the excitation spectra. However, at the same wavelength, the contribution of PSII vibrational fluorescence emission will not be negligible. The spectra of the two preparations were normalized to the respective optical densities of their $\mathrm{Q}_{\mathrm{y}}$ band. The observed conservation of the amount of red pigments absorbing in the region $700-710 \mathrm{~nm}$, confirms that the amount of PSI does not vary among the membranes, as PSII pigments do not absorb to a significant extent at these red wavelengths. The $\mathrm{Q}_{\mathrm{y}}$ excitation maxima of the native and unstacked thylakoid membranes are observed at 678 and $677 \mathrm{~nm}$, respectively. The unstacked membranes are seen to have a larger excitation energy transfer contribution to PSI from 'bulk' Chl $a$ around

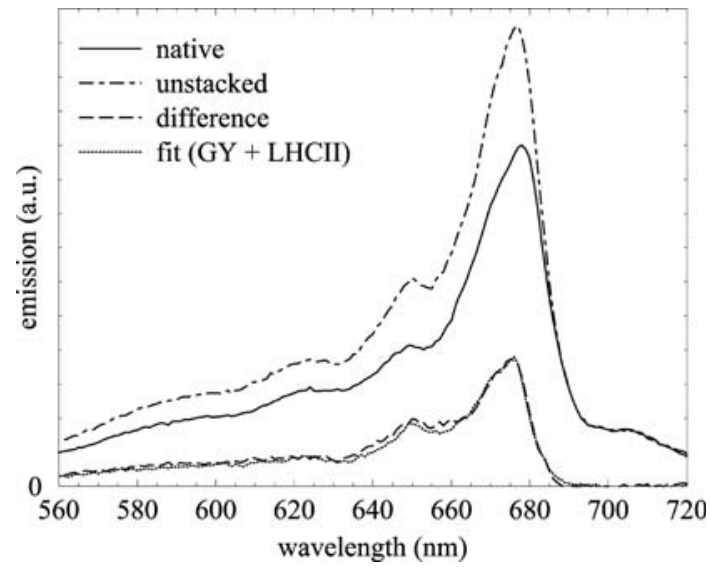

Fig. $277 \mathrm{~K}$ fluorescence excitation spectra of native (solid) and unstacked (dash-dot) thylakoid membranes, normalized to their respective $\mathrm{Q}_{\mathrm{y}}$ absorption maxima. The difference spectrum (unstacked minus native) is plotted (long-dash) along with its fit (dot) consisting of a sum of GY and trimeric LHCII excitation spectra

$675 \mathrm{~nm}$ and $\mathrm{Chl} b$ at $650 \mathrm{~nm}$ with respect to the native thylakoid membranes.

In the same figure, the fluorescence excitation difference spectrum is plotted. This spectrum reveals the origin of the pigments that transfer additional excitation energy to PSI upon unstacking. This spectrum shows peaks at 675 and $650 \mathrm{~nm}$ and a shoulder at $672 \mathrm{~nm}$, and largely resembles the absorption spectrum of trimeric LHCII. However, the excitation difference spectrum extends somewhat to the red with respect to the absorption of trimeric LHCII and shows a higher $\mathrm{Chl} a / b$ ratio. A combination of fluorescence excitation spectra of the PSII core complex, minor Chl $a / b$ complexes and LHCII was necessary to obtain a satisfactory fit, see Fig. 2. For an estimation of the sum of the first two contributions, fluorescence excitation spectra of GY membranes were used, which consist of the PSII core complex and the minor antenna complexes CP29 and CP26 (Ghanotakis et al. 1987). The GY excitation spectrum accounts for absorption strength in the red flank of the excitation difference spectrum (caused by red Chls in the core antenna proteins CP47 and CP43) and has a higher $\mathrm{Chl} a / b$ ratio than LHCII. Because a small amount of PSII fluorescence is recorded at $735 \mathrm{~nm}$ (see, e.g., Andrizhiyevskaya et al. 2005) and the amount of PSII fluorescence is higher in native than in unstacked membranes (Fig. 1), a negative contribution of the PSII absorption is expected in the $735 \mathrm{~nm}$ excitation difference spectrum of unstacked minus native membranes. The positive contribution of the PSII spectrum (from GY) to the excitation difference spectrum therefore evidences the presence of spillover from PSII to PSI in the unstacked thylakoid membranes.

The excitation spectra of the two types of membranes were fitted with a sum of PSI, GY and LHCII excitation 
spectra (normalized to their maximum absorbance in the $\mathrm{Q}_{\mathrm{y}}$ region), resulting in the respective ratios 1:0.19:0.19 in the native membranes and 1:0.36:0.53 in unstacked membranes. We assume that the contributions of GY and LHCII in the excitation spectrum of the native membranes (0.19 each) represent directly excited PSII. This implies that the additional contribution to the fluorescence at $735 \mathrm{~nm}$ in the unstacked membranes is caused by excitation energy transfer from the PSII core (GY) and LHCII to PSI. The additional contribution is larger for LHCII (0.34) than for the PSII core (0.17). We conclude that trimeric LHCII accounts for $2 / 3$ of the fluorescence excitation difference spectrum of unstacked minus native membranes. From the fluorescence excitation spectra in Fig. 2, the PSI antenna is estimated to increase with $\sim 36 \%$ of its Chl $a$ upon unstacking of the thylakoid membrane, of which thus $\sim 24 \%$ is due to the functional association of LHCII with PSI. We conclude that there is a significant extent of LHCII to PSI energy transfer, which could, at least in part, originate from the presence of LHCII-LHCI-PSI supercomplexes in the unstacked thylakoid membranes, as in state 2 membranes (Kouril et al. 2005). The remaining part of the energy transfer to PSI may then originate from spillover to PSI from PSII cores, PSII-LHCII supercomplexes or LHCII that is not directly bound to PSII. Both mechanisms result in a decreased PSII emission and increased PSI emission conform our observations in Fig. 1.

\section{Room temperature time-resolved fluorescence}

To estimate the timescales of these additional energy transfer mechanisms in unstacked with respect to native membranes, time-resolved fluorescence spectra were measured for the membranes at room temperature using a streak camera as detector. In these experiments, the membranes were excited at $485 \mathrm{~nm}$, so as to preferentially excite $\mathrm{Chl} b$. The time-resolution was $28 \mathrm{ps}$ and the PSII reaction centers were kept open using ferricyanide as an electron acceptor. The spectra could be fitted satisfactorily with three fluorescence lifetime components, resulting in the DAS shown in Fig. 3(a-c), which have been scaled to the optical density of the $\mathrm{Q}_{\mathrm{y}}$ absorption maximum of the respective membranes.

A significant difference between the two types of membranes is displayed by the first DAS (Fig. 3a) which has a lifetime of $18 \mathrm{ps}$ in native and $25 \mathrm{ps}$ in unstacked membranes and which has a much larger amplitude in the unstacked membranes. For the native thylakoid membranes, our data show a fluorescence decay as judged from the overall positive amplitude between $665 \mathrm{~nm}$ and $725 \mathrm{~nm}$ with an apparent maximum at $686 \mathrm{~nm}$ (Fig. 3a). One of the two main trapping phases of PSI-LHCI occurs in $20-30$ ps (Ihalainen et al. 2005), originating mainly from excitations in the PSI core complex (Gobets et al. 2001; Gobets and Van Grondelle 2001; Melkozernov et al. 2004). The DAS of this trap peaks around $685 \mathrm{~nm}$ and gradually decreases towards red wavelengths, similar to what we observe for the native membranes. The overall equilibration between LHCI and the PSI core proceeds, however, on longer timescales (Ihalainen et al. 2005). We therefore assign the $18 \mathrm{ps}$ DAS to a mixture of trapping in the PSI core and excitation energy equilibration between LHCI and the PSI core complex. The 18 ps phase of the native membranes seems to lack a significant contribution of PSII. This is consistent with an organization of PSII in larger complexes than cores in these conditions, since PSII cores have a major trapping time of about 40 ps (Miloslavina et al. 2006), whereas trapping in PSII membranes occurs on a longer time-scale (Broess et al. 2006). The DAS of the unstacked membranes, extends to the blue with respect to the native DAS, with its maximum at $680 \mathrm{~nm}$, but moreover it shows a small rise of fluorescence around $730 \mathrm{~nm}$. Energy transfer from LHCII trimers to other complexes is expected to occur on this time-range (Barzda et al. 2001; Van Amerongen and Van Grondelle 2001) as well. Thus, the spectrum and amplitude of the 25 ps energy transfer component in the unstacked membranes with the positive feature at $675-680 \mathrm{~nm}$ and the negative feature above $720 \mathrm{~nm}$ and the absence of these characteristics in the native membranes can be explained by energy transfer from LHCII to PSI in presumably LHCII-LHCI-PSI complexes. The overall larger amplitude of the 25 ps phase in unstacked membranes can be explained by more excitation energy going to PSI and by a larger contribution of trapping in PSII, caused by the occurrence of many PSII complexes as cores in unstacked membranes (Dekker et al. 2002).

An overall positive DAS was resolved with similar lifetimes of 158 and 157 ps in native and unstacked thylakoid membranes, respectively (Fig. 3b). Also this phase is much stronger in unstacked membranes than in native membranes. Both PSI and PSII trap excitation energy by charge separation in their reaction centers at this timescale: PSI-LHCI has been shown to have a second main trapping phase of about $100 \mathrm{ps}$ that is characterized by a redshifted spectrum (Ihalainen et al. 2002, 2005), while the overall excitation decay of PSII in PSII membranes is around 150 ps (Van Mieghem et al. 1992; Broess et al. 2006). As the PSI antenna size has increased at the expense of the PSII antenna in unstacked membranes (see Fig. 1), this DAS is expected to be dominated by PSI trapping for the unstacked membranes, whereas PSII trapping will dominate the native DAS. Indeed, the typical PSI emission decay amplitude around $720 \mathrm{~nm}$ is much larger in the unstacked membranes. However, this spectrum also shows a superposed emission decay at $680 \mathrm{~nm}$ of similar ampli- 
Fig. 3 Room temperature DAS of native (solid) and unstacked (dash) thylakoid membranes.

The excitation wavelength was $485 \mathrm{~nm}$. (a) Comparison of the 18 and 25 ps phase (b) The 158 and 157 ps phase and (c) The 541 and 536 components. The DAS are scaled to the maximum absorption in the $\mathrm{Q}_{\mathrm{y}}$ region
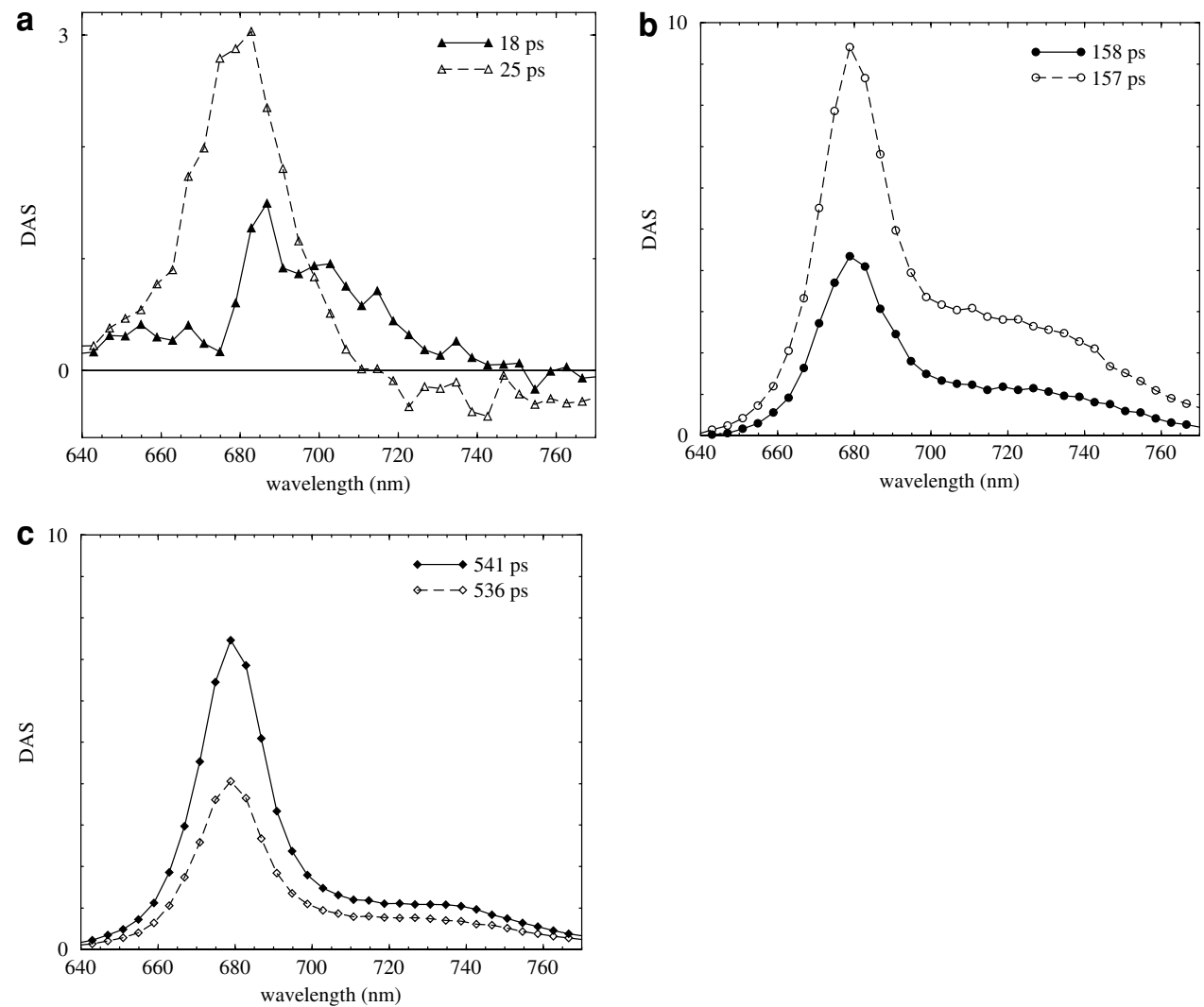

tude as in the native membranes. As the trapping yield will scale with the antenna size, which is smaller in the case of the unstacked membranes, we explain the large contribution at $680 \mathrm{~nm}$ to the $157 \mathrm{ps}$ DAS in the unstacked membranes by the occurrence of LHCII to PSI energy transfer as well as spillover at this timescale. Consequently, the PSI trapping amplitude is partially cancelled by the rise of the emission at the same timescale. Support of this interpretation can be found in the next section. Spillover is expected to lead to a decreased PSII trapping timescale because of the competition between these two processes. Absence of this decrease in decay time upon unstacking of the thylakoid membrane-Fig. 3b-suggests that this decay component is dominated by energy transfer from LHCII to PSI in presumably LHCII-LHCI-PSI supercomplexes rather than spillover.

Trapping at longer timescales was resolved with a lifetime of $541 \mathrm{ps}$ for the native membranes and $536 \mathrm{ps}$ for the unstacked membranes (Fig. 3c). This phase is much stronger in the native membranes. It is known that PSI has trapped all absorbed energy within 120 ps (Ihalainen et al. 2002), whereas the overall excitation decay of PSII in PSII membranes occurs on a timescale of 150 ps (Van Mieghem et al. 1992; Broess et al. 2006). Thus, we assign this long phase to the presence of a fraction of closed PSII reaction centers. If the amount of closed centers is similar in both types of membranes, the contribution of this phase is expected to be higher in the native membranes, because more excitations go to PSII with respect to unstacked membranes (Fig. 1).

The fluorescence kinetics of native and unstacked thylakoid membranes of lettuce, with open PSII reaction centers has been compared by (Hodges et al. 1987). As in our study, they found similar decay times among the two membranes-40, 155, 360, and 863 ps for native membranes and 50, 139, 354, and 1,198 ps for unstacked membranes-which differed significantly in yield. In their case, the main difference in yield existed for the $\sim 150 \mathrm{ps}$ decay, which had a much larger amplitude in case of the unstacked membranes, which is similar to our results (Fig. 3b).

\section{$77 \mathrm{~K}$ time-resolved fluorescence}

To further characterize the energy transfer pathways in native and unstacked membranes, time-resolved fluorescence measurements were performed at $77 \mathrm{~K}$. At this temperature, the fluorescence quantum yield of PSI is relatively high with respect to that of PSII. For proper interpretation of the results, the kinetics of both PSII membranes (BBY preparations) and PSI-LHCI complexes isolated from spinach were measured as well. The excitation wavelength was $485 \mathrm{~nm}$, thus exciting preferentially $\mathrm{Chl} b$. The time-resolution was $10 \mathrm{ps}$. The kinetics of both 
the native and the unstacked thylakoid membranes were satisfactorily fitted with five fluorescence lifetime components. The resulting DAS were normalized to the respective $\mathrm{Q}_{\mathrm{y}}$ absorption maxima of the membranes and are displayed in Fig. $4 a$ and $b$. The kinetics of the BBY membranes and PSI-LHCI could also both be fitted with five components, and the resulting DAS are shown in (Fig. 4c, d). Note that at this low temperature, part of the excitation energy absorbed by PSI-LHCI is trapped on the red pigments and their fluorescence lifetime becomes now dominant for the slow part of the excitation decay in PSI-LHCI.

The native and unstacked membranes, the BBY membranes and the PSI-LHCI complexes all show a sub-10 ps fluorescence lifetime component. In the PSII spectrum (Fig. 4c) this component has a small positive amplitude around $660 \mathrm{~nm}$, which changes into a negative amplitude above $670 \mathrm{~nm}$, a minimum around $680 \mathrm{~nm}$ and about zero amplitude at long wavelengths. This spectral component thus describes energy transfer from Chl $b$ and blue Chl $a$ states to more red Chl $a$ states. In PSI (Fig. 4d), energy equilibration among bulk $(685 \mathrm{~nm})$ and red Chls $(730 \mathrm{~nm})$ is observed to occur on a similar timescale. In this case, the non-conservativity of the DAS is assigned to the trapping of excitations in the PSI core (Gobets et al. 2001; Ihalainen et al. 2002) on early timescales. The PSI spectrum is negative at long wavelengths, which is also observed in the stacked and unstacked thylakoid membranes, indicating that PSI contributes to the sub- 10 ps phase of both. The slightly larger amplitude of the negative contribution around $720 \mathrm{~nm}$ and the positive contribution at $670 \mathrm{~nm}$ in the unstacked membranes suggests that part of the additional PSI antenna transfers the excitation energy on this sub-10 ps timescale to the red states of PSI.

The second estimated decay component, Fig. $4 a$ and b, has a very similar lifetime for both the native and unstacked thylakoid membranes, 39 and $38 \mathrm{ps,} \mathrm{respec-}$ tively, though the spectra differ clearly. For the native membranes, the resolved 39 ps DAS shows great similarity with the second DAS found in BBY samples (Fig. 4c), which has a decay time of $42 \mathrm{ps}$. This DAS describes energy transfer, which is observed to be rather conservative in the BBY membranes, with loss of emission at $675 \mathrm{~nm}$, a zero-crossing at $682 \mathrm{~nm}$ and a gain at $687 \mathrm{~nm}$. This DAS is interpreted as energy transfer from LHCII to the PSII core complex (Andrizhiyevskaya et al. 2005), where the transfer timescale is in agreement with the 32 ps migration time of excitations between LHCII trimers which was estimated at room temperature (Barzda et al. 2001; Van Amerongen and Van Grondelle 2001). The non-conservativity is explained by trapping taking place on a similar timescale in PSII. The $38 \mathrm{ps}$ DAS of the unstacked membranes (Fig. 4b) is seen to have a broadened and larger positive peak at $677 \mathrm{~nm}$ with respect to the $39 \mathrm{ps}$ DAS of the native membranes (Fig. 4a), a zero-crossing at $707 \mathrm{~nm}$ and a
Fig. 477 K DAS of native (a) and unstacked (b) thylakoid membranes which are scaled to their maximum absorption in the $\mathrm{Q}_{\mathrm{y}}$ region, $\mathrm{BBY}$ membranes (c) and isolated PSI-LHCI complexes (d). The excitation wavelength was $485 \mathrm{~nm}$
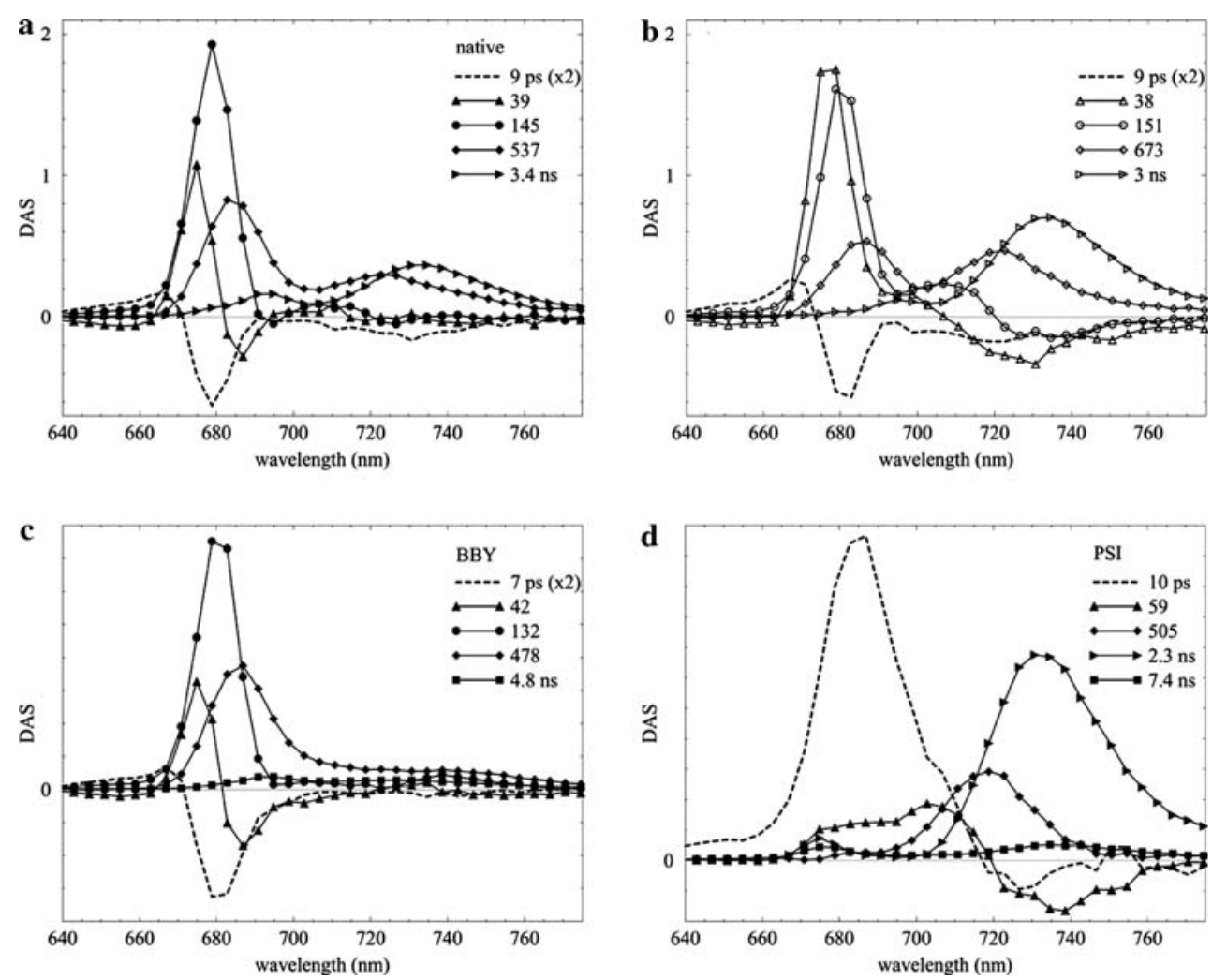
large negative contribution around $730 \mathrm{~nm}$. The latter contribution can be in part explained by the processes within the PSI-LHCI complex (which shows a rather conservative energy transfer spectrum with positive contributions around $700 \mathrm{~nm}$, a zero-crossing at $720 \mathrm{~nm}$ and negative around $740 \mathrm{~nm}-$ Fig. $4 \mathrm{~d}$ ), but the large positive band at $677 \mathrm{~nm}$ (Fig. 4b) does not occur in isolated PSI complexes and the zero-crossing is much further to the blue. We attribute the $38 \mathrm{ps}$ resolved energy transfer component to a new energy transfer process not present in either PSII membranes or PSI complexes, i.e. the main energy transfer component from LHCII to PSI in LHCIILHCI-PSI complexes and subsequent energy equilibration with the red pigments.

In BBY membranes PSII was found to trap excitations on a $132 \mathrm{ps}$ timescale (Fig. 4c, maximum at $681 \mathrm{~nm}$ ). Again, the corresponding DAS of the native membranes (145 ps) shows large spectral similarity with that of BBY, whereas for the unstacked membranes (151 ps) energy transfer from bulk $(681 \mathrm{~nm})$ to red pigments $(730 \mathrm{~nm})$ is found to occur as well. A $150 \mathrm{ps}$ phase appeared to be absent in PSI (Fig. 4d). We explain the 150 ps resolved energy transfer component in the unstacked membranes by energy transfer from LHCII to PSI and partially by spillover from PSII to PSI. The subsequent equilibration (59 ps-Fig. 4d) and trapping (505 ps-Fig. 4d) in PSI explains the observed non-conservativity in the $150 \mathrm{ps}$ DAS of the unstacked membranes. Note that the $680 \mathrm{~nm}$ decay amplitude of this component is only slightly larger for native than for unstacked thylakoid membranes, despite their different PSII antenna size. This suggests that relatively more $680 \mathrm{~nm}$ emission disappears in this time domain, which supports the presence of LHCII to PSI energy transfer and spillover (see also Fig. 3b). Again, there is no decrease in fluorescence decay time upon unstacking of the thylakoid membrane, the timescale even increases from 145 to $151 \mathrm{ps}$, which suggests a dominant contribution of energy transfer from LHCII to LHCI-PSI complexes with respect to spillover to this decay component.

The fourth component has a decay time of $537 \mathrm{ps}$ in case of the native and 673 ps for the unstacked membranes. Both DAS arise as a superposition of the 478 ps PSII trapping in BBY membranes with a spectrum peaking at $687 \mathrm{~nm}$ (Fig. 4c) and the 505 ps trapping in PSI with a spectrum peaking at $718 \mathrm{~nm}$ (Fig. 4c). Clearly, the ratio in which these respective processes contribute differs among the two types of membranes, with a relatively larger PSI contribution in the case of unstacked membranes as seen from the ratio of amplitudes both at 680 and $720 \mathrm{~nm}$. The increased trapping time in unstacked with respect to native membranes may be a consequence of the increased PSI antenna size by the addition of LHCII.
The redmost pigments in PSI, with their fluorescence maximum at $733 \mathrm{~nm}$ (Fig. 4d), decay on both 2.3 and 7.4 ns timescales (Fig. 4d), which is resolved both in the native (3.4 ns) and unstacked membranes ( $3 \mathrm{~ns}$ ) (Fig. 4a and b). These DAS will also cover some slow PSII trapping, though the amplitude of the PSII contribution is probably very small (Fig. 4c). The slow trapping phase in BBY membranes- $4.8 \mathrm{~ns}$-is seen to have its emission maximum at $693 \mathrm{~nm}$ and therefore likely represents trapping on the red pigment(s) of CP47 (Andrizhiyevskaya et al. 2005).

Note that the steady-state emission spectrum arises from the product of the amplitudes and lifetimes of all fluorescence decay phases. For instance, the DAS of BBY (Fig. 4c) show that the band peaking at about $685 \mathrm{~nm}$ has a lifetime of about 500 ps and a large amplitude, whereas the band peaking at about $695 \mathrm{~nm}$ has a lifetime of about $5 \mathrm{~ns}$ but has a much smaller amplitude. This confirms that the two main emission bands of PSII at $77 \mathrm{~K}$ (F-685 and F-695) have very different origins (Andrizhiyevskaya et al. 2005). Similarly, the DAS of PSI-LHCI clearly show the presence of two different pools of red pigments, with emission at 718 and $733 \mathrm{~nm}$, which are respectively located in the core complex and light-harvesting antennae (Van Grondelle et al. 1994). The red pigments of the PSI core are seen to become populated in the early timescale $(9 \mathrm{ps})$, whereas the emission from the red pigments in LHCI arises relatively slowly at the timescale of $59 \mathrm{ps}$ (Fig. 4d).

\section{Conclusion}

Upon cation-depletion, native thylakoid membranes will unstack. We present here a combined study of low temperature fluorescence emission and excitation and show that LHCII is partially released from PSII upon unstacking of the thylakoid membrane and that a part of the LHCII functionally associates with PSI, most likely in the form of LHCII-LHCI-PSI supercomplexes. Time-resolved fluorescence measurements at room temperature reveal energy transfer from LHCII to PSI to take place on a timescale of $25 \mathrm{ps}$, which is somewhat longer at $77 \mathrm{~K}$ (38 ps), consistent with the organization in an LHCII-LHCI-PSI supercomplex. A second estimated timescale for energy transfer from LHCII to PSI-150-160 ps-will originate from energy transfer from LHCII which is less tightly associated with an LHCI-PSI complex than LHCII which transfers energy to LHCI-PSI on a 30 ps timescale, or perhaps from a second LHCII trimer bound to an LHCII-LHCI-PSI supercomplex. Spillover from PSII to PSI was found to take place at the same timescale (150-160 ps) also both at room temperature and $77 \mathrm{~K}$, which is the same timescale 
on which trapping occurs in PSII in intact membranes. Our results suggest that cation depletion induces direct energy transfer from LHCII to PSI and therefore at least partially mimics a state transition.

Acknowledgments We thank I.H.M. van Stokkum for help in global analysis. This work was supported by the Netherlands Organization for Scientific Research (NWO) by a grant from the Foundation for Fundamental Research on Matter (FOM), and by the European Union by a grant to the PSICO Research Training Network (HPRN-CT-2002-00248) and the Intro2 Marie Curie Research Training Network (MRTN-CT-2003-505069).

\section{References}

Albertsson PÅ, Andreasson E (2004) The constant proportion of grana and stroma lamellae in plant chloroplasts. Physiol Plant 121:334-342

Allen JF, Forsberg J (2001) Molecular recognition in thylakoid structure and function. Trends Plant Sci 6:317-326

Andrizhiyevskaya EG, Chojnicka A, Bautista JA, Diner BA, Van Grondelle R, Dekker JP (2005) Origin of the F685 and F695 fluorescence in photosystem II. Photosynth Res 84:173-180

Andrizhiyevskaya EG, Schwabe TME, Germano M, D'Haene S, Kruip J, Van Grondelle R, Dekker JP (2002) Spectroscopic properties of PSI-IsiA supercomplexes from the cyanobacterium Synechococcus PCC 7942. Biochim Biophys Acta 1556:265-272

Barber J (1980) An explanation for the relationship between salt-induced thylakoid stacking and the chlorophyll fluorescence changes associated with changes in spillover of energy from photosystem II to photosystem I. FEBS Lett 118:1-10

Barzda V, Gulbinas V, Kananavicius R, Van Amerongen H, Van Grondelle R, Valkunas L (2001) Singlet-singlet annihilation kinetics in aggregates and trimers of LHCII. Biophys J 80:24092421

Ben-Shem A, Frolow F, Nelson N (2003) Crystal structure of plant photosystem I. Nature 426:630-635

Ben-Shem A, Frolow F, Nelson N (2004) Evolution of photosystem I-from symmetry through pseudosymmetry to asymmetry. FEBS Lett 564:274-280

Berthold DA, Babcock GT, Yocum CF (1981) A highly resolved, oxygen-evolving photosystem II preparation from spinach thylakoid membranes: EPR and electron-transport properties. FEBS Lett 134:231-234

Briantais JM, Vernotte C, Olive J, Wollman FA (1984) Kinetics of cation-induced changes of photosystem II fluorescence and of lateral distribution of the two photosystems in the thylakoid membranes of pea chloroplasts. Biochim Biophys Acta 766:1-8

Broess K, Trinkunas G, Van der Weij-de Wit CD, Dekker JP, Van Hoek A, Van Amerongen H (2006) Excitation energy transfer and charge separation in photosystem II membranes revisited. Biophysical J 91:3776-3786

Dekker JP, Boekema EJ (2005) Supramolecular organization of thylakoid membrane proteins in green plants. Biochim Biophys Acta 1706:12-39

Dekker JP, Germano M, Van Roon H, Boekema EJ (2002) Photosystem II solubilizes as a monomer by mild detergent treatment of unstacked thylakoid membranes. Photosynth Res 72:203-210

Depège N, Bellafiore S, Rochaix JD (2003) Role of chloroplast protein kinase Stt7 in LHCII phosphorylation and state transition in Chlamydomonas. Science 299:1572-1575

Ghanotakis DF, Demetriou DM, Yocum CF (1987) Isolation and characterization of an oxygen-evolving photosystem-II reaction center core preparation and a $28 \mathrm{kDa}$ chl-alpha-binding protein. Biochim Biophys Acta 891:15-21

Gobets B, Van Grondelle R (2001) Energy transfer and trapping in photosystem I. Biochim Biophys Acta 1507:80-99

Gobets B, Van Stokkum IHM, Rögner M, Kruip J, Schlodder E, Karapetyan NV, Dekker JP, Van Grondelle R (2001) Time-resolved fluorescence emission measurements of photosystem I particles of various cyanobacteria: a unified compartment model. Biophys J 81:407-424

Hodges M, Briantias JM, Moya I (1987) The effect of thylakoid membrane reorganization on chlorophyll fluorescence lifetime components: a comparison between state transitions, protein phosphorylation and the absence of $\mathrm{Mg}^{2+}$. Biochim Biophys Acta 893:480-489

Holzwarth AR (1996) Data analysis of time-resolved measurements. In: Amesz J, Hoff AJ (eds) Biophysical techniques in photosynthesis. Advances in photosynthesis and respiration, vol 3. Kluwer Academic Publishers, Dordrecht, The Netherlands, pp $75-92$

Ihalainen JA, Jensen PE, Haldrup A, Van Stokkum IHM, Van Grondelle R, Scheller HV, Dekker JP (2002) Pigment organization and energy transfer dynamics in isolated Photosystem I (PSI) complexes from Arabidopsis thaliana depleted of the PSIG, PSI-K, PSI-L, or PSI-N subunit. Biophys J 83:2190-2201

Ihalainen JA, Klimmek F, Ganeteg U, Van Stokkum IHM, Van Grondelle R, Jansson S, Dekker JP (2005) Excitation energy trapping in photosystem I complexes depleted in Lhcal and Lhca4. FEBS Lett 579:4787-4791

Jensen PE, Haldrup A, Zhang S, Scheller HV (2004) The PSI-O subunit of plant photosystem I is involved in balancing the excitation pressure between the two photosystems. J Biol Chem 279:24212-24217

Kirchhoff H, Borinsky M, Lehnert S, Chi LF, Büchel C (2004) Transversal and lateral exciton energy transfer in grana thylakoids of spinach. Biochemistry 43:14508-14516

Klimmek F, Ganeteg U, Ihalainen JA, Van Roon H, Jensen PE, Scheller HV, Dekker JP, Jansson S (2005) Structure of higher plant light-harvesting complex I-in vivo characterization and structural interdependence of the Lhca proteins. Biochemistry 44:3065-3073

Kouril R, Zygadlo A, Arteni AA, De Wit CD, Dekker JP, Jensen PE, Scheller HV, Boekema EJ (2005) Structural characterization of a complex of photosystem I and light-harvesting complex II of Arabidopsis thaliana. Biochemistry 44:10935-10940

Leibl W, Breton J, Deprez J, Trissl H-W (1989) Photoelectric study on the kinetics of trapping and charge stabilization in oriented PSII-membranes. Photosynth Res 22:257-275

Liu Z, Yan H, Wang K, Kuang T, Zhang J, Gui L (2004) Crystal structure of spinach major light-harvesting complex at $2.72 \AA$ resolution. Nature 428:287-292

Lunde C, Jensen PE, Haldrup A, Knoetzel J, Scheller HV (2000) The PSI-H subunit of photosystem I is essential for state transitions in plant photosynthesis. Nature 408:613-615

Melkozernov AN, Kargul J, Lin S, Barber J, Blankenship RE (2004) Energy-coupling in the PSI-LHCI supercomplex from the green alga Chlamydomonas reindhartii. J Phys Chem B 108:1054710555

Miloslavina Y, Szczepaniak M, Muller MG, Sander J, Nowaczyk M, Rogner M, Holzwarth AR (2006) Charge separation kinetics in intact photosystem II core particles is trap-limited. A picosecond fluorescence study. Biochemistry 45:2436-2442

Murata N (1969) Control of excitation transfer in photosynthesis. Light induced change of chlorophyll a fluorescence in Porphyridium cruentum. Biochim Biophys Acta 172:242-251

Palacios MA, De Weerd FL, Ihalainen JA, Van Grondelle R, Van Amerongen H (2002) Superradiance and exciton (de)localization 
in light-harvesting complex II from green plants? J Phys Chem B 106:5782-5787

Stoitchkova K, Busheva M, Apostolova E, Andreeva A (2006) Changes in the energy distribution in mutant thylakoid membranes of pea with modified pigment content. II. Changes due to magnesium ions concentration. J Photochem Photobiol B $83: 11-20$

Van Amerongen H, Van Grondelle R (2001) Understanding the energy transfer function of LHCII, the major light-harvesting complex of green plants. J Phys Chem B 105:604-617

Van Grondelle R, Dekker JP, Gillbro T, Sundström V (1994) Energy transfer and trapping in photosynthesis. Biochim Biophys Acta 1187:1-65

Van Leeuwen PJ, Nieveen MC, Van de Meent EJ, Dekker JP, Van Gorkom HJ (1991) Rapid and simple isolation of pure photosystem II core and reaction center particles from spinach. Photosynth Res 28:149-153
Van Mieghem FJE, Searle GFW, Rutherford AW, Schaafsma TJ (1992) The influence of the double reduction of $Q_{A}$ on the fluorescence decay kinetics of photosystem II. Biochim Biophys Acta 1100:198-206

Van Stokkum IHM, Larsen DS, Van Grondelle R (2004) Global and target analysis of time-resolved spectra. Biochim Biophys Acta 1657:82-104

Williams RS, Allen JF, Brain APR, Ellis RJ (1987) Effect of $\mathrm{Mg}^{2+}$ on excitation energy transfer between LHCII and LHCI in a chlorophyll-protein complex. FEBS Lett 225:59-66

Wollman FA (2001) State-transitions reveal the dynamics and flexibility of the photosynthetic apparatus. EMBO J 20:36233630

Zhang S, Scheller HV (2004) Light-harvesting complex II binds to several small subunits of photosystem I. J Bio Chem 279:3180 3187 